\title{
Kölner Dermatologische Gesellschaft
}

\section{Sitzung vom 30. Januar 1931}

Falkenstcin: Lichen ruber. Unterarme, Mundschleimhaut und Penis sind befallen. Kein Jucken. Aus der Universitätshatäkünik.

Zinsser: Verruccae planae lichenoides. Lichen-ruber-ähnliche Warzen-bildungen auf Unterarmen und Gesicht.

Zinsser: TJlcus genitocruralis. (Wiedervorstellung.) Die Geschwüre sind sämtlich abgeheilt. Zinsser: ülkus des Fußrückens. (Wiedervorstellung.) 1·Tachdem aus ge-wissen Gründen artifizieller Ursprung der Ulzeration vermutet werden konnte, heilte das jahrelang bestehende Geschwür unter Okklusivverbänden in kurzer .Zeit ab.

Zinsser: Narbige Alopezie der Augenbrauen und des Kopfes. Der Zustand stellt den Rest einer Triphaldermatitis dar.

Zinsser: Psoriasis. Vorstellung von 8 Fallen der verschiedensten Formen von Psoriasis. Der Versuch der Behandlung mit Psorimangan ist noch nicht abgeschlossen. Im Anschluß an die Vorstellung entspinnt sich eine Debatte über Psoriasisbehandlung.

Krantz: Mikrosporie beim Erwachsenen. Infektion durch seine an Mikro-sporie erkrankten Kinder.

Sitzung vom 28. Februar 1931.

Dreyer: Herpes zoster der Mundschleimhaut. Gruppierte grau belegte, von rotem Hof umgebene Erosionen auf Lippe, Zunge und Wangenschleimhaut. Schmerzhafte Schwellung der Unterkieferdrüzen. Die Affektion ist plötzlich entstanden. Zinsser bezweifelt die Diagnose des Fehlens der Einseitigkeit wegen und meint, daß es sich auch um aphthöse Mundentzündung handeln kann.

Dreyer: Fall zur Diagnose. Psoriatiker weist auf der Kopfhaut zahlreiche rote kreis- und girlandenförmige Herde mit erhabenen ßändern auf, die stark jucken. Psoriasis wird nicht angenommen, während Zinsser doch diese Diagnose stellen möchte.

Dreyer: Ekzem und Vitiligo. Um einen neurodermiticchen Herd am Ober-schenkel ist die Haut vitiliginös. Auch am Skrotum Vitiligostellen.

Falkenstein: Lupus der Ohrmuschel. Einziger Herd auf der Rückseite des linken Ohres seit einem Jahr bestehend. Keine nachweisbare Tuberkulose.

Aus der Universitätshautklinik.

Zinsser: Salvarsandermatitis. 10 Tage nach kombinierter Bi-Sa-Kur Auf-treten einer mit starken Infiltraten einhergehenden Dermatitis der Gesäßhaut, die sich schnell über den Körper ausbreitete.

Zinsser: Salvarsandermatitis. Schwere Dermatitis, aufgetreten 8 Wochen nach der letzten Salvarsaninjektion.

Zinsser: Salvarsanresistente Syphilis. Primäraffekt vor eineinhalb Jahren. Er blieb neben anderen spirochätenhaltigen Ulzera zirka dreiviertel Jahr be-stehen, trotz der Behandlung. Dann dauernd Rezidive, die nur schwer auf Salvarsan-, Bismut-, Quecksilber-, Jod- und Zittmannbehandlung wichen. Auch 
182 Гcriodische L·iteratur.

jetzt wieder zahlreiche großpapulöse Herde vom Typ der sekundären Spät-syphilide. Modlmayr berichtet über einen Patienten mit Nasengumma, das der Salvarsan-, Bismut- und

Jodkalibehandlung trotzte und erst auf Merzinol-injektionen zur Heilung kam.

Zinsser: Ekzem mit Eiereiiveißüberempfinälichkeit. Seit 9 Jahren leidet

die 24 jährige Patientin an Ekzem des Gesichts, der Arme und des Halses.

Auf die Anlegung von 2 Quaddeln mit unverdünntem Hühnereiweiß erfolgte

ein bedrohlich aussehender Urtikariaausbruch. Merkliche Besserung setzte im

Anschluß hieran ein. Modlmayr und Stern schlagen die Desensibilisierung mit starken Verdünnungen vor. Fritz Falkensiein-Köln.

Periodische Literatur.

Zusammengestellt von Dr. Arthur Alexander in Charlottenburg.

Dermatologie.

AUgemeine Pathologic Pathologische Anatomic.

Kreibich, C, Prag, Eruption oder Reaktion. Derm. Wscbr. ßd. 91. Nr. 47.

Buschke hat der traumatisehen Reaktion wieder breiteren Raum gegeben

und läßt durch das Trauma nicht nur banale Scheuerungsphänomene, sondern

auch spezifische Hautveränderungen entstehen. Kr. führt den Buschkeschew

Gedanken welter fort an Hand seiner alten Hypothese, daß systematisierte

Veränderungen Reaktionen des Sympathikus auf indirekte Reize darstellen,

Eruption ist das Entstehen von Effloreszenzen ohne äußeres Zutun, Reaktion

auf äußere Reize hin. Auf die Schädlichkeit kann reagieren 1. das Endothel,.

2. der Gefäßnervenapparat, und 3. kann eine zerebrale Beeinflussung des peri-

pheren Apparates vorliegen. Das vegetative Nervensystem arbeitet im Auftrage

des zerebrospinalen. Bei dieser Hypothese werden Eruptionen zu Reaktionen.

Zum Beispiel ist der Zoster klinisch Eruption, nach obiger Hypothese aber

Reaktion (auf Reize vom Ganglion, Endemien usw.). Dabei können 2 Fälle

eintreten: 1. der Reiz ist so groß, daß die Reaktion ohne äußeres Zutun als

Eruption erscheint, oder 2. reicht der Reiz nur aus, eine Metamere zu sensi-

bilisieren, worauf erst äußei'e Reize die Reaktion auslösen. Zu dieser Theorie

gibt Kr. eine ganze Reihe klinischer Beispiele. Schlockermann-Berlio.

Urbach, Orale Desensibilisierung alimentär-idiosynkrasischer Dermatosen mittels artspezifischer Peptone. Klin. $\Lambda$ Vschr. Nr. 44. 1930.

Verf. sieht in den alimentären Idiosynkrasien eine viel häufigere Ursache von Dermatosen als im allgemeinen angenommen wird, und zwar nicht nur der akuten toxischen Exantheme, sondern auch der Urtikaria, des Prurigo, des Strofulus usw. Mit Hilfe des sogenannten

Ernährungsexperimentes geiiingt es • ihm in vielen Fallen, die schädliche Substanz zu erkennen, während die intra-kutane Testprüfung sich in praxi als unbrauchbar erwiesen hat. Es gelang ihm in leichteren und erst kürzere Zeit bestehenden Fallen von alimentärer Idio-synkrasie durch etwa 14tägige völlige Eiweißentziehung mit nachfolgender langsamer Eiweißzufuhr oftmals

Dauerheilung zu erzielen. Bei schwereren Formen bewährten sich die artspezifischen

Peptonvormahlzeiten (Propeptone) auf das beste. Sie wirkten nicht nur temporal'

streptophylaktisch, sondern führten bei systematischer Einnahme auch zu dauernder

Desensibilisierung. Der 